E17-2005-140

Ž. Lj. Kovačević ${ }^{1,2, a}$, V.S. Oudovenko ${ }^{1,3, b}$

\title{
PAULI SPIN SUSCEPTIBILITY IN THE $t-J$ MODEL
}

Submitted to «Theoretical and Mathematical Physics»

${ }^{1}$ Bogoliubov Laboratory of Theoretical Physics, Joint Institute for Nuclear Research, 141980 Dubna, Russia

${ }^{2}$ Faculty of Natural Sciences and Mathematics, University of Montenegro, P.O. Box 211, 81000 Podgorica, Serbia and Montenegro

${ }^{3}$ Center for Materials Theory, Department of Physics and Astronomy, Rutgers University, Piscataway, NJ 08854, USA

${ }^{a}$ E-mail: zarkok@cg.ac.yu

${ }^{b}$ E-mail: udovenko@theor.jinr.ru 
Ковачевич Ж.Л., Удовенко В.С.

E17-2005-140

Спиновая восприимчивость Паули в $t-J$ модели

Вычислена зависимость спиновой восприимчивости от концентрации носителей на основе самосогласованной теории для функций Грина в технике операторов Хаббарда.

Посвящается 25-летию основания факультета естественных наук и математики Университета Черногории (Подгорица).

Работа выполнена в Лаборатории теоретической физики им. Н. Н. Боголюбова ОИЯИ.

Препринт Объединенного института ядерных исследований. Дубна, 2005

Kovačević Ž. Lj., Oudovenko V.S.

E17-2005-140

Pauli Spin Susceptibility in the $t-J$ Model

Using a self-consistent theory for the Green functions of Hubbard operators, the spin susceptibility is calculated as a function of the carrier concentration.

Dedicated to the 25th anniversary of the Faculty of Natural Sciences and Mathematics, University of Montenegro, Podgorica.

The investigation has been performed at the Bogoliubov Laboratory of Theoretical Physics, JINR. 


\section{INTRODUCTION}

The problem of $\mathrm{CuO}_{2}$ ground state in high-temperature superconducting cuprate compounds or how to introduce the quasiparticles in such a way that the calculated physical quantities behave as ones obtained in the experimental measurements is still open. A classification of cuprate unusual physical properties leads to a generic $(T, p)$ phase diagram, in terms of temperature $T$ and the hole concentration $p$ in the $\mathrm{CuO}_{2}$ planes [1].

Present investigation is devoted to investigation of the magnetic susceptibility $\chi$. Many different techniques have been proposed to research spin response of strongly correlated electron systems with one of those being $\mathrm{CuO}_{2}$ plane $[1,2]$.

One of the main questions in constructing the theory of HTSC is about the correct form for the dynamical spin susceptibility $\chi(\mathbf{q}, \omega)$. For $\omega=0$, static $\mathbf{q}$ dependent spin susceptibility has two limits: spatially uniform limit with $\mathbf{q} \rightarrow 0$ and staggered one with $\mathbf{q} \rightarrow(\pi, \pi)$, which is the antiferromagnetic (AF) wave vector [1].

A special interest presents the investigation of the static and uniform susceptibility $\chi=\chi(0,0)$. It could be measured as macroscopic susceptibility $\chi_{m}$ [3-9] or indirectly through Knight shift nuclear magnetic resonance (NMR) technique $[10,11]$ or through specific heat measurements [13-16]. Also measuring of the temperature $T^{*}$ of the maximum value of static spin susceptibility is very important [8, 10, 11 and 17-18].

Concerning the experimental results of the hole doped $\mathrm{La}_{2-x} \mathrm{Sr}_{x} \mathrm{CuO}_{4-y}$ (LSCO) and $\mathrm{YBa}_{2} \mathrm{C}_{3} \mathrm{O}_{6+x}$ (YBCO) family parent compounds, one meets difficulties due to the different procedure of sample preparation. Since the value of the correlation length $\xi$ of the AF fluctuation is of the range of the interatomic distance, therefore considered strongly correlated many-particle system is very sensitive to some short-range structural disorder, i. e. structural defects such as impurities and vacancies [1]. In order to obtain intrinsic spin susceptibility of hole doped $\mathrm{CuO}_{2}$ planes, one has to subtract hole-doping independent part in LSCO [4] and Curie-like $\left(C_{g} / T\right)$ paramagnetic contribution, which characterizes isolated impurities and defects in YBCO compounds [2-5].

The static uniform spin susceptibility was calculated in the framework of the Hubbard [19-24], Heisenberg [25] and $t-J$ [26-28] models. There was found qualitative agreement between theoretical and experimental results for temperature and doping dependencies. The self-consistent renormalization theory of spin 
fluctuations [21] describes well experimental results for the staggered part of the uniform spin susceptibility.

Among the theoretical investigations aimed at obtaining full form of $\chi(\mathbf{q}, \omega)$, one should notice the semiphenomenological Millis-Monien-Pines (MMP) theory [29-33], which was successfully applied for description of many experimental findings. Also there are a lot of attempts to obtain $\chi(\mathbf{q}, \omega)$ microscopically. Very often microscopical findings use either the method of equations of motion for Green functions (GF) [34-38] or Mori's memory function method [39-43]. Early theoretical efforts were directed to find some universal form or scaling function which connects $\chi, \chi_{\max }, T^{*}$ and vacancy or impurity concentration $x[2,4$, 44-46 and 47] for the high-temperature limit. Using the Van Hove scenario, some theories were able to qualitatively explain dependencies $\chi$ on $x$ and $T$ [23, 49-52]. Numerical cluster calculations in the framework of $t-J$ or two-band Hubbard model [53-55] had success in obtaining form of the dependencies of the chemical potential $\mu$ on hole concentration and $T$ similar to the ones obtained in [56-61].

In the $\mathrm{CuO}_{2}$ planes of $\mathrm{LSCO}$ compounds for low $\mathrm{Sr}$ content, carriers are holes, while for large $x$ they are electrons [7]. It was confirmed with corresponding change of Hall coefficient sign from positive to negative one and with the doping evolution of the Fermi surface form [1,62], as also was obtained from ARPES experiments [58-61] and ED calculations [53-55].

In the proposition that the chemical potential is close to the Van Hove singularity in the quasiparticle DOS, a lot of different DOS models were considered [23, 48-52]. There was obtained satisfactory description of the experimental results of the temperature dependence macroscopic spin susceptibility $[48,23]$ or Knight shift [49, 51, 26].

In the NMR Knight shift experiments [10,11], one comes to conclusion that there is no evidence for Van Hove singularity in DOS. There were established linear relations between spin susceptibility $\chi_{m}$ and respectively ${ }^{89} \mathrm{Y}$ Knight shift in YBCO [10] and ${ }^{63} \mathrm{Cu}$ Knight shift in LSCO [11] compounds. There was also found monotonic behaviour of $\chi(x)$ on doping $x$. However, one should note that the samples were not in the overdoped phase and also that during the NMR measurements they were influenced by high-magnetic fields (well above $10 \mathrm{~T}$ ).

Recently in Ref. [62], a band-structure of the $t-J$ model in the normal and superconducting states of the paramagnetic phase was profoundly investigated. We extend the research in this paper taking the external magnetic field into consideration. The main goal of the present investigation is to derive the corresponding expression for $\chi=\chi(0,0)$ in the $t-J$ model with the correct band-dispersion energy spectrum [62], to get which the interaction between quasiparticles is included through the renormalization of their energies caused by AF spin fluctuations. Renormalization of quasiparticle energy also changes the uniform and static spin susceptibility. 
Our paper is organized as follows. In the next section, we write the $t-J$ model using the Hubbard operators formalism and obtain the corresponding Green function (GF). In Sec.2, we calculate the energy-dispersion relations, just as it was done in Ref. [62]. Section 3 is devoted to calculation of the static and uniform spin susceptibility. Section 4 contains numerical results and discussions. Our conclusions can be found in Sec. 5 .

\section{MODEL HAMILTONIAN AND GREEN FUNCTION}

We intend to calculate the spin response of a strongly correlated electron system to a uniform magnetic field. So, we consider an effective $t-J$ model for the $\mathrm{CuO}_{2}$ plane perturbated with the Zeeman interaction

$$
H_{t-J}^{h}=H_{t-J}+H_{h}
$$

with the $t-J$ Hamiltonian written in the standard notation $[1,62]$

$$
H_{t-J}=\sum_{\langle i, j\rangle, \sigma} t_{i j}\left(1-n_{i \bar{\sigma}}\right) c_{i \sigma}^{+} c_{j \sigma}\left(1-n_{j \bar{\sigma}}\right)+\sum_{\langle i, j\rangle} J_{i j}\left(\mathbf{S}_{i} \mathbf{S}_{j}-\frac{1}{4} n_{i} n_{j}\right),
$$

where $\bar{\sigma}=-\sigma$ and the Zeeman's Hamiltonian in the external magnetic field $\mathbf{h}=(0,0, h)$ is

$$
H_{h}=-h \sum_{i} \mu_{i}^{z}
$$

where $\mu_{i}^{z}=-g \mu_{B} S_{i}^{z}$ is the $z$ component of magnetic moment with gyromagnetic (Landé) factor $g$ and Bohr magneton $\mu_{B}$. In the Hubbard operator representation $X_{i}^{\alpha \beta}=|i \alpha\rangle\langle i \beta|$ which preserves site no double occupancy constraint, i. e. $X_{i}^{00}+$ $\sum_{\sigma} X_{i}^{\sigma \sigma}=1$, one has

$$
n_{i}=\sum_{\sigma} X_{i}^{\sigma \sigma}, \quad S_{i}^{z}=\frac{1}{2} \sigma\left(n_{i \sigma}-n_{i \bar{\sigma}}\right)=\frac{1}{2} \sum_{\sigma} \sigma X_{i}^{\sigma \sigma}, \quad X_{i}^{\sigma 0}=c_{i \sigma}^{+}\left(1-n_{i \bar{\sigma}}\right) .
$$

Considered Hamiltonian (1) takes the following form [62]:

$$
H_{t-J}^{h}=\sum_{i \sigma} \xi_{i \sigma}^{h} X_{i}^{\sigma \sigma}+\sum_{i \neq j, \sigma} t_{i j} X_{i}^{\sigma 0} X_{j}^{0 \sigma}+\frac{1}{4} \sum_{i \neq j, \sigma} J_{i j}\left(X_{i}^{\sigma \bar{\sigma}} X_{j}^{\bar{\sigma} \sigma}-X_{i}^{\sigma \sigma} X_{j}^{\bar{\sigma} \bar{\sigma}}\right),
$$

where $\xi_{i \sigma}^{h}=\varepsilon_{d \sigma}^{h}-\mu$ and $\varepsilon_{d \sigma}^{h}=\varepsilon_{d}+\mu_{B} h \sigma$ are the energy of the hole with spin $\sigma$ in the magnetic field $h$. The exchange integral for the the nearest neighbors (n.n.) is $J_{i j}=J$ and zero in other cases, $t_{i j}=t$ and $t_{i j}=t^{\prime}$ are respectively 
the hopping parameters for the n.n. and the next to the nearest neighbors (n.n.n.) sites $i, j$ on a two-dimensional (2D) square lattice and $\mu$ is the chemical potential.

The main goal of the present investigation is calculation of the static spin susceptibility $\chi$ for strongly correlated electronic system in a uniform static magnetic field $\mathbf{h}=(0,0, h)$. Using the definition of the average magnetic moment or magnetization as $M_{z}^{h}=\mathcal{N}\left\langle\mu_{z}^{h}\right\rangle$, or equivalently $M_{z}^{h}=-\partial\left\langle H_{t-J}^{h}\right\rangle / \partial h$, where $\mathcal{N}$ is number of the $2 \mathrm{D}$ lattice site, one has the spin susceptibility as the zero field limit of derivation $\chi=\left(\partial M_{z}^{h} / \partial h\right)_{h \rightarrow 0}$.

As one can see, to obtain static spin susceptibility $\chi$, one should first calculate spin-dependent on-site occupation number $n_{i \sigma}^{h}$ which is

$$
n_{i \sigma}^{h}=\left\langle X_{i}^{\sigma \sigma}\right\rangle^{h}=\left\langle X_{i}^{\sigma 0} X_{i}^{0 \sigma}\right\rangle^{h},
$$

i. e. one should calculate the following anticommutator Green function:

$$
\left\langle\left\langle X_{i}^{0 \sigma}(t) ; X_{j}^{\sigma 0}\left(t^{\prime}\right)\right\rangle\right\rangle=-i \Theta\left(t-t^{\prime}\right)\left\langle\left[X_{i}^{0 \sigma}(t), X_{j}^{\sigma 0}\left(t^{\prime}\right)\right]_{+}\right\rangle,
$$

where we have chosen Zubarev's general formulation [63].

\section{QUASIPARTICLE DISPERSION}

As analytical tools, we use the projection method in the GF equation of motion $[62,63]$

$$
\omega\left\langle\left\langle X_{i}^{0 \sigma} \mid X_{j}^{\sigma 0}\right\rangle\right\rangle_{\omega}=\left\langle\left[X_{i}^{0 \sigma}, X_{j}^{\sigma 0}\right]_{+}\right\rangle+\left\langle\left\langle Z_{i \sigma} \mid X_{j}^{\sigma 0}\right\rangle\right\rangle_{\omega},
$$

where $\left\langle\left\langle X_{i}^{0 \sigma} \mid X_{j}^{\sigma 0}\right\rangle\right\rangle_{\omega}$ is the Fourier transform of GF (7), the anticommutator of the operators $A$ and $B$ is $[A, B]_{+}=A B+B A$ and the equilibrium average value of an operator $A$ is defined as $\langle A\rangle=\operatorname{Tr}\left\{\exp \left(-\beta H_{t-J}\right) A\right\}$ with canonical partition function (or «Zustandssumme») as trace (Tr) in the energy representation of the operator $\exp \left(-\beta H_{t-J}\right)$. In the projection method one extracts the main linear part after scattering in the equation of motion for transition described with $X_{i}^{0 \sigma}$, which can be analytically described with the following procedure [62]:

$$
Z_{i \sigma}=\left[X_{i}^{0 \sigma}, H_{t-J}^{h}\right]=\sum_{l} E_{i l \sigma}^{h} X_{l}^{0 \sigma}+Z_{i \sigma}^{i r} .
$$

From the projection condition $\left\langle\left[Z_{i \sigma}^{i r}, X_{j}^{\sigma 0}\right]_{+}\right\rangle=0$, one obtains the «frequency matrix»

$$
E_{i j \sigma}^{h}=\left\langle\left[\left[X_{i}^{0 \sigma}, H_{t-J}^{h}\right], X_{j}^{\sigma 0}\right]_{+}\right\rangle / Q,
$$

whose spatial and temporal Fourier transformation will give energy band dispersion $E_{\mathbf{q}}(\omega)$. In the equation of motion for the Hubbard operator $X_{i}^{0 \sigma}$

$$
\left(i \frac{d}{d t}-\xi_{i \sigma}^{h}\right) X_{i}^{0 \sigma}=-\sum_{l \sigma^{\prime}} t_{i l} B_{i \sigma \sigma^{\prime}} X_{l}^{0 \sigma^{\prime}}+\frac{1}{2} \sum_{l \sigma^{\prime}} J_{i l}\left(B_{l \sigma \sigma^{\prime}}-\delta_{\sigma \sigma^{\prime}}\right) X_{i}^{0 \sigma^{\prime}},
$$


appear Bose-like Hubbard operators [62]

$$
B_{i \sigma \sigma^{\prime}}=Q_{i}^{\sigma} \delta_{\sigma \sigma^{\prime}}+X_{i}^{\bar{\sigma} \sigma^{\prime}},
$$

which describes hole scattering on charge (or kinematics interactions)

$$
Q_{i}^{\sigma}=X_{i}^{00}+X_{i}^{\sigma \sigma}=1-n_{i} / 2+\sigma S_{i}^{z},
$$

and spin fluctuations (or exchange interactions $X_{i}^{\bar{\sigma} \sigma}=S_{i}^{\bar{\sigma}}$. Like in Hubbard I approximation, we further neglect density-density charge fluctuations but we keep spin correlations introducing spin correlation functions for the n.n. $\chi_{1 s}=$ $\left\langle\mathbf{S}_{i} \mathbf{S}_{i+\mathbf{a}_{1}}\right\rangle$ and for the n.n.n. lattice sites $\chi_{2 s}=\left\langle\mathbf{S}_{i} \mathbf{S}_{i+\mathbf{a}_{2}}\right\rangle$, where n.n. describe $\mathbf{a}_{1}=\left( \pm a_{x}, \pm a_{y}\right)$ and n.n.n. describe $\mathbf{a}_{2}= \pm\left(a_{x} \pm a_{y}\right)$ radius vectors. For the paramagnetic phase, we have $\left\langle S_{i}^{z}\right\rangle=0$ and also $\left\langle Q_{i}^{\sigma}\right\rangle=\left\langle Q_{i}^{\bar{\sigma}}\right\rangle \equiv Q=$ $1-n / 2$, where $n=\left\langle n_{i}\right\rangle=\mathcal{N}^{-1} \sum_{i \sigma}\left\langle X_{i}^{\sigma \sigma}\right\rangle$ is the average hole on-site occupation number. Using the common representation for the spin products $\mathbf{S}_{l} \mathbf{S}_{j}=S_{l}^{z} S_{j}^{z}+$ $\frac{1}{2} \sum_{\sigma} S_{l}^{\sigma} S_{j}^{\bar{\sigma}}$, one has

$$
\left\langle B_{l \sigma \sigma} Q_{j}^{\sigma}+B_{l \sigma \bar{\sigma}} X_{j}^{\sigma \bar{\sigma}}\right\rangle=\left\langle\mathbf{S}_{l} \mathbf{S}_{j}\right\rangle+1-\left\langle n_{j}\right\rangle+\left\langle n_{l}\right\rangle\left\langle n_{j}\right\rangle / 4 .
$$

In such a way, we obtain the following elements of the «frequency matrix»:

$$
E_{i j \sigma}^{h}=\delta_{i j}\left(\xi_{i \sigma}^{h}+\delta \mu\right)+\left(1-\delta_{i j}\right) \tilde{E}_{i j \sigma},
$$

with diagonal and

$\xi_{i \sigma}^{h}+\delta \mu=\left\{\xi_{i \sigma}^{h} Q+\sum_{l} t_{i l}\left\langle X_{i}^{\bar{\sigma} 0} X_{l}^{0 \bar{\sigma}}\right\rangle+\frac{1}{2} \sum_{l} J_{i l}\left(\left\langle\mathbf{S}_{l} \mathbf{S}_{i}\right\rangle-\frac{1}{2}\left\langle n_{j}\right\rangle+\frac{1}{4}\left\langle n_{l}\right\rangle\left\langle n_{j}\right\rangle\right)\right\} / Q$,

next to them

$$
\tilde{E}_{i j \sigma}=\left\{t_{i j}\left(\left\langle\mathbf{S}_{i} \mathbf{S}_{j}\right\rangle+1-\left\langle n_{j}\right\rangle+\frac{1}{4}\left\langle n_{i}\right\rangle\left\langle n_{j}\right\rangle\right)+\frac{1}{2} J_{i j}\left\langle X_{j}^{\bar{\sigma} 0} X_{i}^{0 \bar{\sigma}}\right\rangle\right\} / Q
$$

matrix elements. Further we perform the spatial Fourier transformations of the upper correlation functions

$$
\begin{aligned}
& \left\langle X_{i}^{\bar{\sigma} 0} X_{l}^{0 \bar{\sigma}}\right\rangle=\frac{1}{\mathcal{N}} \sum_{\mathbf{k}} n_{\mathbf{k} \bar{\sigma}} e^{i \mathbf{k} \mathbf{R}_{i l}}, \quad n_{\mathbf{k} \bar{\sigma}}=\left\langle X_{\mathbf{k}}^{\bar{\sigma} 0} X_{-\mathbf{k}}^{0 \bar{\sigma}}\right\rangle, \\
& n_{i \sigma}=\left\langle X_{i}^{\sigma \sigma}\right\rangle=\left\langle X_{i}^{\sigma 0} X_{i}^{0 \sigma}\right\rangle=\frac{1}{\mathcal{N}} \sum_{\mathbf{k}} n_{\mathbf{k} \sigma}
\end{aligned}
$$


and

$$
\sum_{l} t_{i l}\left\langle X_{i}^{\bar{\sigma} 0} X_{l}^{0 \bar{\sigma}}\right\rangle=\frac{1}{\mathcal{N}} \sum_{\mathbf{k}} t(\mathbf{k}) n_{\mathbf{k} \bar{\sigma}}, \quad t(\mathbf{k})=4 t \gamma(\mathbf{k})+4 t^{\prime} \gamma^{\prime}(\mathbf{k})
$$

with $\gamma(\mathbf{q})=\left[\cos \left(a_{x} q_{x}\right)+\cos \left(a_{y} q_{y}\right)\right] / 2$ and $\gamma^{\prime}(\mathbf{q})=\cos \left(a_{x} q_{x}\right) \cos \left(a_{y} q_{y}\right)$. As we consider only n.n. exchange interactions, we have

$$
\frac{1}{2} \sum_{l} J_{i l}\left\langle\mathbf{S}_{l} \mathbf{S}_{i}\right\rangle=\frac{1}{2} J \sum_{\mathbf{a}_{1}}\left\langle\mathbf{S}_{i} \mathbf{S}_{i+\mathbf{a}_{1}}\right\rangle=2 J \chi_{1 s} .
$$

In $\mathbf{q}$ representation one obtains the following GF:

$$
\left\langle\left\langle X^{0 \sigma} \mid X^{\sigma 0}\right\rangle\right\rangle_{\mathbf{q}, \omega}^{h}=\frac{Q}{\omega-E_{\mathbf{q} \sigma}^{h}}=\frac{Q}{\omega-\left(\tilde{E}_{\mathbf{q} \sigma}+\tilde{\xi}_{i \sigma}^{h}\right)} .
$$

As in [62], the quasiparticle energy $\tilde{E}_{\mathbf{k} \sigma}$ is renormalized due to the kinematic $\epsilon(\mathbf{k})=t(\mathbf{k})$, bonding between it and exchange $\epsilon_{s}(\mathbf{k})=4 t \gamma(\mathbf{k}) \chi_{1 s}+4 t^{\prime} \gamma^{\prime}(\mathbf{k}) \chi_{2 s}$, and the net exchange interaction $(\propto J)$ as follows:

$$
\tilde{E}_{\mathbf{k} \sigma}=-Q \epsilon(\mathbf{k})-\frac{\epsilon_{s}(\mathbf{k})}{Q}-\frac{2 J}{\mathcal{N}} \sum_{\mathbf{q}} \frac{\gamma(\mathbf{k}-\mathbf{q})}{Q} n_{\mathbf{q} \bar{\sigma}} .
$$

Within the model used in [62], the static spin correlation functions which appear in the definition of $\epsilon_{s}(\mathbf{k})$ are respectively

$$
\chi_{1 s}=\left\langle\mathbf{S}_{i} \mathbf{S}_{i+a_{1}}\right\rangle=\frac{1}{\mathcal{N}} \sum_{\mathbf{q}} \gamma(\mathbf{q})\left\langle\mathbf{S}_{\mathbf{q}} \mathbf{S}_{-\mathbf{q}}\right\rangle
$$

and

$$
\chi_{2 s}=\left\langle\mathbf{S}_{i} \mathbf{S}_{i+a_{2}}\right\rangle=\frac{1}{\mathcal{N}} \sum_{\mathbf{q}} \gamma^{\prime}(\mathbf{q})\left\langle\mathbf{S}_{\mathbf{q}} \mathbf{S}_{-\mathbf{q}}\right\rangle,
$$

where $\left\langle\mathbf{S}_{\mathbf{q}} \mathbf{S}_{-\mathbf{q}}\right\rangle=\left(\pi \omega_{s} / 2\right) \chi_{s}(q)$. Here $\mathbf{q}$-dependent part of spin-fluctuation susceptibility [62] is

$$
\chi_{s}(q)=\frac{\chi_{0}}{1+\xi^{2}[1+\gamma(q)]},
$$

where $\xi$ is the characteristic AF correlation length, spin-fluctuation energy is $\omega_{s} \simeq J$ and $\chi_{0}$ is some normalization parameter [62].

The renormalized one-particle on-site spin-dependent energies are

$$
\tilde{\xi}_{i \sigma}^{h}=\xi_{i \sigma}^{h}+\delta \mu_{\sigma},
$$


where the chemical potential shift is

$$
\delta \mu_{\sigma}=\frac{1}{\mathcal{N}} \sum_{\mathbf{q}} \frac{t(\mathbf{q})}{Q} n_{\mathbf{q} \bar{\sigma}}-\frac{2 J}{Q}\left(\frac{1}{2}\left\langle n_{j}\right\rangle-\chi_{1 s}-\frac{1}{4}\left\langle n_{l}\right\rangle\left\langle n_{j}\right\rangle\right) .
$$

In the upper formulae we should take $n_{\mathbf{q} \bar{\sigma}}=n_{\mathbf{q} \sigma} \equiv n_{\mathbf{q}}$, which means that $\tilde{E}_{\mathbf{k} \sigma}=\tilde{E}_{\mathbf{k} \bar{\sigma}} \equiv \tilde{E}_{\mathbf{k}}$ and $\delta \mu_{\sigma}=\delta \mu_{\bar{\sigma}} \equiv \delta \mu$, since these quantities relate to the paramagnetic phase and the external magnetic field is absent.

\section{QUASIPARTICLE SPIN SUSCEPTIBILITY}

As one can see in GF (21), the only dependence on magnetic field $h$ and on the spin direction $\sigma$ is contained in the on-site hole energy $\xi_{i \sigma}^{h}$. This means that in the lowest order, the effect of an external static uniform magnetic field is to shift the relative energy distributions of the up and down spins. So we have different on-site occupation number for spin up and down, but our many-particle system of holes will reach an equilibrium in such a manner that their Fermi levels (or the renormalized chemical potential $\mu-\delta \mu_{\sigma}$ ) are the same.

For on-site spin- and field-dependent occupation numbers, we have

$$
\begin{aligned}
n_{\sigma}^{h}=\left\langle X_{i}^{\sigma \sigma}\right\rangle^{h}=\left\langle X_{i}^{\sigma 0} X_{i}^{0 \sigma}\right\rangle^{h}= & \frac{1}{\mathcal{N}} \sum_{\mathbf{k}}\left\langle X^{\sigma 0} X^{0 \sigma}\right\rangle_{\mathbf{k}}^{h}= \\
& =\frac{1}{\mathcal{N}} \sum_{\mathbf{k}} \frac{1}{2 \pi} \int_{-\infty}^{+\infty} d \omega\left\langle X^{\sigma 0} X^{0 \sigma}\right\rangle_{\mathbf{k}, \omega}^{h}
\end{aligned}
$$

i. e. according to the general receipt of the GF spectral representation [63], we have

$$
\begin{aligned}
& n_{\sigma}^{h}=\frac{1}{\mathcal{N}} \sum_{\mathbf{k}} \frac{1}{2 \pi} \int_{-\infty}^{+\infty} d \omega \frac{-2}{e^{\beta \omega}+1} \operatorname{Im}\left\langle\left\langle X^{\sigma 0} \mid X^{0 \sigma}\right\rangle\right\rangle_{\mathbf{k}, \omega}^{h}= \\
&=\frac{1}{\mathcal{N}} \sum_{\mathbf{k}} \int_{-\infty}^{+\infty} d \omega \frac{Q}{e^{\beta \omega}+1} \delta\left(\omega-\tilde{E}_{\mathbf{k}}^{h}\right),
\end{aligned}
$$

with $\beta=1 /\left(k_{B} T\right)$. In such a way, we obtain in the magnetic field $h$ the following expression for the spin $\sigma$ dependent on-site occupancy:

$$
n_{\sigma}^{h}=\frac{1}{\mathcal{N}} \sum_{\mathbf{k}} n_{\mathbf{k} \sigma}^{h}=\frac{1}{\mathcal{N}} \sum_{\mathbf{k}} Q /\left\{1+\exp \left[\beta\left(\tilde{E}_{\mathbf{k}}+\tilde{\xi}_{i \sigma}^{h}\right)\right]\right\}
$$


and the magnetization in a uniform magnetic field $h$ is $M^{h}=-g \mu_{B} \mathcal{N}\left(n_{\uparrow}^{h}-\right.$ $\left.n_{\downarrow}^{h}\right) / 2$. Further one should calculate derivative on $h$ of the $M^{h}$ to obtain the uniform and static susceptibility

$$
\chi_{0}^{h}=\left(\frac{\partial M^{h}}{\partial h}\right)=\mathcal{N} g \mu_{B} \frac{\partial}{\partial h}\left(n_{\downarrow}^{h}-n_{\uparrow}^{h}\right) / 2 .
$$

Making the derivative on $h$ of the $n_{\sigma}^{h}$ one gets

$$
\frac{\partial n_{\sigma}^{h}}{\partial h}=\frac{1}{\mathcal{N}} \frac{-\sigma g \mu_{B}}{k_{B} T} Q \sum_{\mathbf{k}} \frac{\exp \left[\beta\left(\tilde{E}_{\mathbf{k}}+\tilde{\xi}_{i \sigma}^{h}\right)\right]}{\left\{1+\exp \left[\beta\left(\tilde{E}_{\mathbf{k}}+\tilde{\xi}_{i \sigma}^{h}\right)\right]\right\}^{2}},
$$

which, including (29) and (30), gives the following expression for the uniform and static spin susceptibility in the uniform magnetic field $\mathbf{h}=(0,0, h)$ in a paramagnetic phase:

$$
\chi_{0}^{h}=\frac{g \mu_{B}^{2}}{k_{B} T} \sum_{\mathbf{k} \sigma}\left[n_{\mathbf{k} \sigma}^{h}-\left(n_{\mathbf{k} \sigma}^{h}\right)^{2} / Q\right],
$$

with

$$
n_{\mathbf{k} \sigma}^{h}-\left(n_{\mathbf{k} \sigma}^{h}\right)^{2} / Q=\frac{\beta Q}{2[1+\cosh (\beta x)]}=\frac{\beta Q}{\left(1+e^{\beta x}\right)\left(1+e^{-\beta x}\right)},
$$

where $x=\tilde{E}_{\mathbf{k}}+\delta \mu-\mu+\mu_{\sigma} h \sigma+\varepsilon_{d}$ and where spin $\sigma$ and field $h$ dependent on-site hole occupancy is given in Eq. (29).

For a large sample $(\mathcal{N} \rightarrow \infty)$, one can turn to the upper expressions on the continual limit $\frac{1}{\mathcal{N}} \sum_{\mathbf{k}} \ldots \Longrightarrow \int_{1 \text { st BZ }} \ldots d \mathbf{k} /(2 \pi)^{2}$, where one integrates over the first Brillouin zone (1st BZ). In the zero-field limit, one finds the static and uniform spin susceptibility in the paramagnetic phase

$$
\begin{aligned}
\chi_{0}=\left(2 \mu_{B}\right)^{2} \int_{1 \mathrm{st} \mathrm{BZ}} \frac{d \mathbf{k}}{(2 \pi)^{2}}\left(-\frac{d n_{\mathbf{k}}}{d \tilde{E}_{\mathbf{k}}}\right) & =\left(2 \mu_{B}\right)^{2} \\
& \int_{1 \mathrm{st} \mathrm{BZ}} \frac{d \mathbf{k}}{(2 \pi)^{2}}\left(\frac{d n_{\mathbf{k}}}{d \mu}\right)=\left(2 \mu_{B}\right)^{2} \frac{d}{d \mu} N,
\end{aligned}
$$

where we use $g \simeq 2$. Total number of the holes (depending on $\mathrm{CuO}_{2}$ plane hole doping), which in canonical ensemble fixes the Fermi-level position, is given by

$$
\frac{1}{2} N=\int_{1 \mathrm{st} \mathrm{BZ}} \frac{d \mathbf{k}}{(2 \pi)^{2}} n_{\mathbf{k}}=\int_{1 \mathrm{st} \mathrm{BZ}} \frac{d \mathbf{k}}{(2 \pi)^{2}} Q /\left\{1+\exp \left[\beta\left(\tilde{E}_{\mathbf{k}}+\delta \mu-\mu\right)\right]\right\},
$$

where the quasiparticle energy $\tilde{E}_{\mathbf{k}}$ is given by (22) and the chemical potential shift is given by (26), taking into account the remark bellow Eq. (26). 


\section{NUMERICAL RESULTS AND DISCUSSION}

In this section, we present numerical results, namely the chemical potential $\mu(p)$, uniform static quasiparticle spin susceptibility $\chi_{0}(p)$ (at zero and finite temperatures) and the energy dispersion and FS shape evolution with hole doping $p$. To perform the numerical calculations, we have chosen the following parameter values: $t^{\prime}=-0.3$ and $J=0.4$. The n.n. hoping parameter $t$ was chosen as an energy unit. In order to get the self-consistent solution, a $64 \times 64$ cluster was used. The energy mesh was less than 0.001 .

In Fig. 1, we plot dependencies $\mu(p)$ and $\chi_{0}(p)$ at two temperatures $T=0$ and $T=0.3 t>1000 \mathrm{~K}$. The zero temperature dependencies of the chemical potential (solid lines) and the susceptibility (dot-dashed line) are linear functions on the hole doping. We obtain that $\mu$ does not depend on $T$ in the considered doping interval. For small hole doping $p<0.05$, one can see only weak decrease

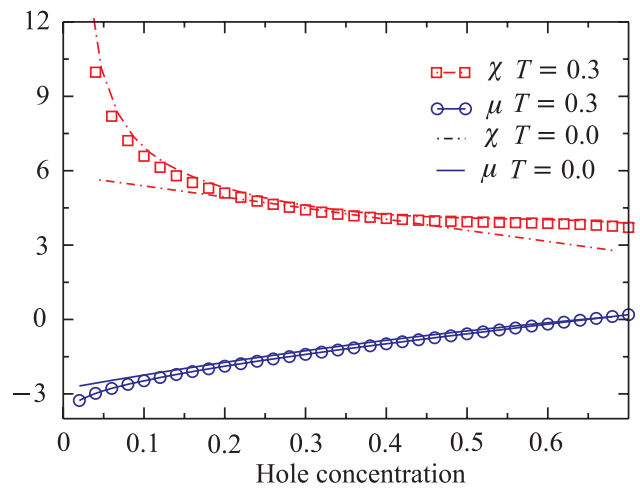

Fig. 1. Doping dependence of the chemical potential $\mu$ at temperature $T=0$ (solid line) and $T=0.3$ (in units of $t$ ) (solid line with circles) are plotted in the figure. The susceptibility, $\chi$ vs doping at $T=0$ is plotted by dot-dashed line and $T=0.3$ case is showed by dot-dashed line with squares. The susceptibility $\chi$ is in units of $\left(2 \mu_{B}\right)^{2}$

of $\mu$ as $T$ increase from 0 to $0.3 t$. In the ED numerical studies [53,54] in the hole overdoped phase $(0.15<p)$, it was obtained that $\mu$ does not depend on $T$. Under assumption that the chemical potential is $T$-independent and that DOS peak is narrow with rectangular shape [48], one successfully describes many of the normal state properties of HTSC compounds on qualitative level. It gives some support that our result about weak temperature dependence of $\mu$ is correct.

One of the basic features which follows from the experiments is an increase of the macroscopic $\chi_{m}$ in the hole underdoped phase and $\chi_{m}$ decrease in the hole overdoped phase, which is better seen in the LSCO compounds $[6,7,48]$ than in the YBCO ones $[3,5]$, where the overdoping phase is very short. Existence 
of the maxima in the $\chi_{m}(p)$ dependence can be concluded from Sommerfeld's $\gamma$ parameter dependence on $p$ in the electronic specific heat measurements $[1$, 13-16] and also from the numerical results obtained in the framework of the Hubbard model [55]. However, in some experiments performed in YBCO [10,11] and LSCO [11,12] compounds, one finds a linear scaling between NMR Knight shifts ${ }^{89} \mathrm{~K}$ (or ${ }^{63} \mathrm{~K}$ ) in the magnetic field of about $H=10 \mathrm{~T}$ and $\chi_{m}$ at about $H=1 \mathrm{~T}$ and also that Knight shifts are monotonously increasing functions on hole doping $p$. In other words, there we do not find maxima in the $K(p)$ and $\chi(p)$ dependencies. Generally, one should be careful extracting inherent characteristics of the quasiparticles in the $\mathrm{CuO}_{2}$ without oxygen vacancy and different inhomogeneity contributions. From our numerical results, it follows that $\chi_{0}$ decreases with hole doping $p$ increase which is in agreement with theoretical results [28] for $p>0.05$ (where our approximations are available for paramagnetic phase).

Looking after the experimental results about $\chi_{m}$ with maxima depending on $p$, one can conclude that our numerical results for $\chi(p)$ and $\mu(p)$ have the form that one could expect for hole overdoped phase.

On the other hand, the staggered part $\chi_{\mathbf{Q}}$ of the static spin susceptibility decreases and shifts to the higher energies upon hole doping, see for example $[1,2,20,26]$. Uniform static quasiparticle part $\chi_{0}$ even get enhanced with Stoner exchange and is approximately $T$-independent $[19,21]$, which is in agreement with our results presented in Fig. 1. As it follows from results of Ref. [21], the origin of the temperature dependence of $\chi_{m}$ is the effect of the AF spin fluctuations, which is described by $\chi_{\mathbf{Q}}$. The same conclusion also comes from results of investigations of the relaxation of a rare-earth impurity ion, where one can explain the $T$ dependence of the crystal field linewidth as an effect of the spin fluctuations, described with the staggered spin susceptibility $\chi_{\mathbf{Q}}[64-$ 68]. Although we should notice that there is also another point of view that $\chi_{\mathbf{Q}}$ describes charge density wave instability of the ground state [26]. In our equation for uniform spin susceptibility (34), influence of the AF spin fluctuations is included in the corresponding renormalization of the quasiparticle energy spectrum $\tilde{E}_{\mathbf{q}}$ given by (22). So one can understand strong increase of $\chi_{0}$ at low hole doping and in the high-temperature regime, presented in Fig. 1 as the effect of the AF spin fluctuations.

In Figs. 2-4, we present evolution of the Fermi surface (FS) and the quasiparticle energy dispersion on the hole doping. One can see on the top contour plots transformation of the 2D FS from four pocket-like centered at $M(\pi, \pi)$ points of the BZ for small doping $p<0.5$ to the electron-like FS centered at $\Gamma(0,0)$ point of BZ for large doping $p>0.5$. However, from the experimental results [59], FS shape transforms at $p=0.22$, similar to the numerical results (for $p<0.3$ ) in the framework of the Hubbard model [55]. This point could indicate that $t-J$ model is not an adequate model for quantitative description of real materials. 


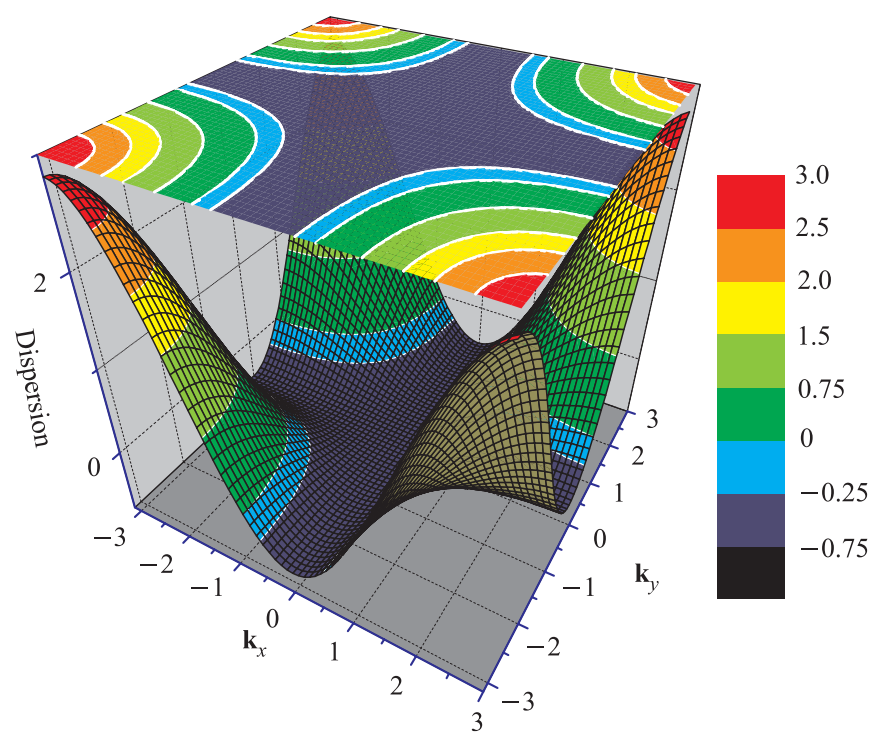

Fig. 2. 3D and contour plot of the hole dispersion is plotted for hole concentration 0.3

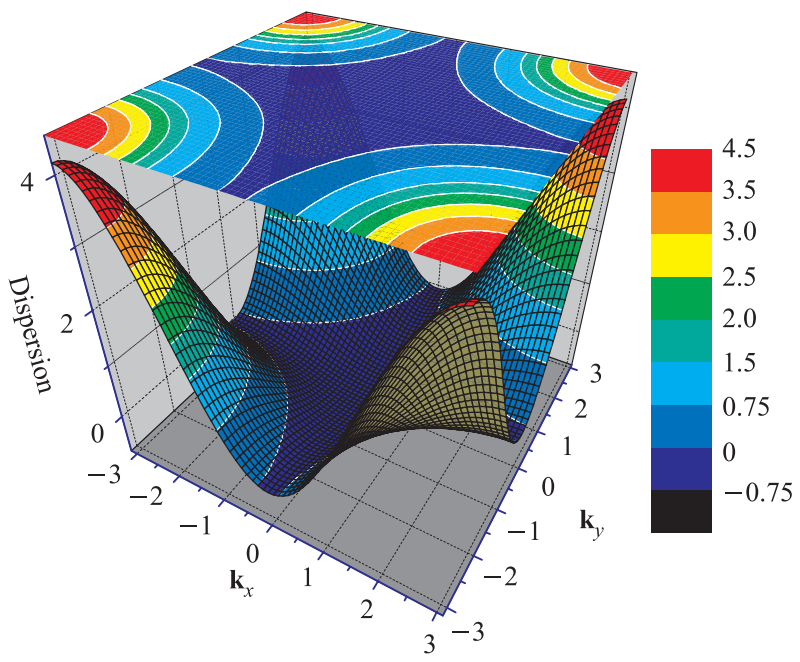

Fig. 3. 3D and contour plot of the hole dispersion is plotted for hole concentration 0.5 


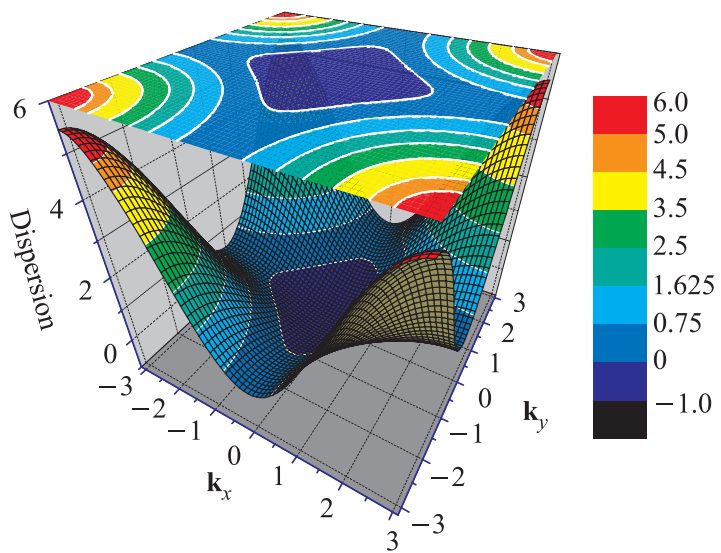

Fig. 4. 3D and contour plot of the hole dispersion is plotted for hole concentration 0.7

\section{CONCLUSIONS}

In the framework of $t-J$ model, we have investigated hole concentration dependencies of the chemical potential $\mu$ and static spatially uniform spin susceptibility $\chi_{0}$ in the $\mathrm{CuO}_{2}$ plane, the common block of the HTSC cuprate compounds. We used the projection method technique of the GF equations of motion. In the considered case, Pauli-like itinerant susceptibility presents inherent quasiparticle part of the magnetic response. Antiferromagnetic fluctuations and strong correlations were included in the mean-field approximation method used to obtain corresponding renormalization of the quasiparticle energies. For small doping at high temperature, contribution of the AF fluctuations became dominant, which led to strong increase of the quasiparticle magnetic response.

Evolution of the FS shape has no essential effect on the inherent quasiparticle uniform static susceptibility. One could expect that FS transformation will lead to a strong influence on the staggered part of magnetic response, which can explain temperature and doping dependencies of the static spin susceptibility. However, in order to fit the experimental results better, one should repair hole concentration dependencies obtained in the framework of the $t-J$ model probably using the Hubbard model as more adequate one for the description of high-temperature superconductors [69].

Acknowledgements. Ž. K. would like to thank the Joint Institute for Nuclear Research for the hospitality. We gratefully acknowledge stimulating discussions with Prof. N. M. Plakida and possibility to use his book [1], prior the publication. 


\section{REFERENCES}

1. Plakida N.M. High-Temperature Superconductivity. Experiment, Theory and Aplication. 2nd edition. Berlin: Springer-Verlag (in press).

2. Johnston D.C. Normal-state magnetic properties of single-layer cuprate hightemperature superconductors and related materials // Handbook of Magnetic Materials. Amsterdam, 1997. V. 10.

3. Johnston D. C. et al. // Physica C. 1988. V.153-155. P.572-577.

4. Johnston D. C. // Phys. Rev. Lett. 1989. V.62. P. 957.

5. Tranquada J. M. et al. // Phys. Rev. B. 1988. V.38. P. 2477.

6. Takagi H. et al. // Phys. Rev. B. 1989. V.40. P. 2254.

7. Torrance J. B. et al. // Phys. Rev. B. 1989. V.40. P. 8872.

8. Allgeier C., Schilling J. S. // Phys. Rev. B. 1993. V.48. P. 9747.

9. Kondo T. et al. // Phys. Rev. B. 1994. V.50. P. 1244.

10. Alloul H., Ohno T., Mendels P. // Phys. Rev. Lett. 1989. V.63. P. 1700.

11. Song Y.-Q. et al. // Phys. Rev. Lett. 1993. V.70. P.3131.

12. Ohsugi Sh., Kitaoka Y., Asayama K. // Physica C. 1997. V. 282-287. P. 1373-1374.

13. Loram J. W. et al. // Physica C. 2000. V.341-348. P. 831.

14. Loram J. W. et al. // J. Phys. Chem. Solids. 2001. V.62. P. 59-64.

15. Tallon J. L. et al. // Physica C. 2004. V.415. P.9-14.

16. Matsuzaki T. et al. // J. Phys. Soc. Jpn. 2004. V.73. P. 2232.

17. Hwang H. Y. et al. // Phys. Rev. Lett. 1994. V.72. P. 2636.

18. Timusk T., Statt B. // Rep. Prog. Phys. 1999. V.62. P. 61-122

19. Bulut N. et al. // Phys. Rev. B. 1990. V.41. P. 1797.

20. Letz M., Sigmund E., Mehring M. // Phys. Lett. A. 1995. V. 197. P. 67-72.

21. Ping L. // J. Phys.: Condens. Matter. 1995. V.7. P. 5351-5358.

22. Trapper U., Ihle D., Fehske H. // Phys. Rev. B. 1996. V.54. P. 7614.

23. Eremin M. V., Solovianov S. G., Varlamov S. V. // ZhETF. 1997. V.112. P.17631777. 
24. Calegari E.J., Magalhães S. G., Gomes A. A. // Int. J. Mod. Phys. B. 2002. V. 16. P. 3895-3907.

25. Siurakshina L., Ihle D., Hayn R. // Phys. Rev. B. 2000. V.61. P. 14601.

26. Eremin I. et al. // Phys. Rev. B. 1997. V.56. P. 11305.

27. Sherman A., Schreiber M. // Mod. Phys. Lett. B. 2003. V. 17. P.433-440.

28. Sherman A., Schreiber M. // Eur. Phys. J. B. 2003. V. 32. P. 203-214.

29. Millis A. J., Monien H., Pines D. // Phys. Rev. B. 1990. V.42. P. 167.

30. Monien H., Pines D., Takigawa M. // Phys. Rev. B. 1991. V. 43. P. 258.

31. Monien H., Monthoux P., Pines D. // Phys. Rev. B. 1991. V.43. P. 275.

32. Millis A. J., Monien H. // Phys. Rev. B. 1992. V. 45. P. 3059.

33. Brzykin V., Pines D., Thelen // Phys. Rev. B. 1994. V.50. P. 16052.

34. Shimahara H., Takada S. // J. Phys. Soc. Jpn. 1991. V.60. P. 2394.

35. Shimahara H., Takada S. // J. Phys. Soc. Jpn. 1992. V.61. P. 989.

36. Yushankhai V. Yu., Hayn R., Ihle D. JINR Preprint E 17-96-17. Dubna, 1996.

37. Zavidonov A. Yu., Brinkmann D. // Phys. Rev. B. 1998. V.58. P. 12486.

38. Winterfeldt S., Ihle D. // Phys. Rev. B. 1998. V.58. P. 9402.

39. Tserkovnikov Yu.A. // TMF. 1982. V. 52. P. 147-160 (in Russian); Theor. and Math. Phys. 1983. V.52. P.712.

40. Jackeli G., Plakida N. M. // TMF. 1998. V.114. P. 426.

41. Pantić M. R. // Int. J. Mod. Phys. B. 2002. V. 16. P. 4743.

42. Sega I., Prelovšek P., Bonča J. // Phys. Rev. B. 2003. V.68. P. 054524.

43. Vladimirov A. A., Ihle D., Plakida N. M. // TMF. 2005 (submitted).

44. Levin G. A., Quader K. F. // Phys. Rev. B. 1996. V.53. P. R530.

45. Levin G. A., Quader K. F. // Physica C. 1996. V. 258. P. 261-272.

46. Nakano T. et al. // Phys. Rev. B. 1994. V.49. P. 16000.

47. Singh R. R. P., Glenister R. L. // Phys. Rev. B. 1992. V.46. P. 11871.

48. Moshchalkov V. V. Physica B. 1990. V. 163. P. 59-62.

49. Aristov D. N., Yashenkin A. G. // Physica C. 1995. V. 248. P. 22-28. 
50. Thoma J., Tewari S., Ruvalds J., Rieck C. T. // Phys. Rev. B. 1995. V.51. P. 15393.

51. Bok J., Bouvier J. // Physica C. 1995. V.255. P. 357-360.

52. Bok J., Bouvier J. // J. Supercond. 2000. V.13. P.781-787.

53. Jaklič J., Prelovšek P. // Phys. Rev. Lett. 1996. V.77. P. 892.

54. Jaklič J., Prelovšek P. // Adv. Phys. 2000. V. 49. P. 1-92.

55. Mancini F., Avella A. // Adv. Phys. 2004. V.53. P. 537.

56. Dagotto E. et al. // Phys. Rev. B. 1992. V.45. P. 10741.

57. Izyumov Yu.A., Letfulov B. M., Shipitsyn E.V. // J. Phys.: Condens. Matter. 1994. V.6. P. 5137-5154.

58. Ino A. et al. // Phys. Rev. Lett. 1997. V.79. P. 2101.

59. Ino A. et al. arXiv:cond-mat/0005370v2.

60. Prelovšek P., Ramšak A. // Phys. Rev. B. 2002. V.65. P. 174529.

61. Harima N. et al. // Phys. Rev. B. 2003. V.67. P. 172501.

62. Plakida N. M., Oudovenko V. S. // Phys. Rev. B. 1999. V.59. P. 11949.

63. Zubarev D. N. // Usp. Fiz. Nauk. 1960. V.71. P.71. (in Russian); Sov. Phys. Usp. 1960. V. 3. P. 320.

64. Goremychkin E. A., Osborn R., Taylor A.D. // Pis'ma Zh. Eksp. Teor. Fiz. 1989. V.50. P.351. (in Russian); JETP Lett. 1989. V.50. P. 380.

65. Osborn R., Goremychkin E.A. // Physica C. 1991. V. 185-189. P. 1179.

66. Kovačević Ž., Plakida N. M. // Pis'ma Zh. Eksp. Teor. Fiz. 1993. V. 57. P. 238; JETP Lett. 1993. V.57. P. 249.

67. Kovačević Ž., Plakida N. M. // Physica C. 1994. V. 235-240. P. 1685.

68. Kovačević Ž., Plakida N. M. // Physica C. 1994. V. 228. P. 15.

69. Plakida N.M., Hayn R., Richard J.-L. // Phys. Rev. B. 1995. V.51. P. 16599. 


\section{Редактор Н. С. Скокова}

Подписано в печать 27.10.2005.

Формат $60 \times 90 / 16$. Бумага офсетная. Печать офсетная.

Усл. печ. л. 1,1. Уч.-изд. л. 1,5. Тираж 305 экз. Заказ № 55076.

Издательский отдел Объединенного института ядерных исследований 141980, г. Дубна, Московская обл., ул. Жолио-Кюри, 6.

E-mail: publish@pds.jinr.ru www.jinr.ru/publish/ 